\title{
İlerleyen Yanma Dalgalarının Evans Fonksiyonu İle Spektral Kararlılı̆̆ı
}

\author{
Fatih ÖZBAĞ $\breve{1}^{*}$, Burcu CEYLAN KURU²
}

\section{$\ddot{\mathbf{O z}}$}

Bu çalışmada başlangıçta bir miktar yakıt içeren gözenekli ortama hava enjekte edilerek geliştirilmiş petrol geri kazanımı modelinde ortaya çıkan yanma dalgaları incelenmiştir. Yanma modeli sıcaklık, oksijen ve yakıt olmak üzere üç bağımlı değişkenden oluşmaktadır. Sistemde sıcaklık ve oksijenin hızı aynı kabul edilip ters yönde ilerleyen yanma dalgaları göz önünde bulundurulmuştur. Modelimiz ters yönde ilerleyen bir yanma dalgası üzerinde linerize edilmiş ve linerize edilmiş bu operatörün esas spektrumu incelenmiştir. Spektral kararlılığın sağlanması için esas spektrumu sol yarı düzleme taşıyan bir ağılık fonksiyonu elde edilmiştir. Ayrık spektrumu bulabilmek için Evans fonksiyonu kullanılmışır. Sağ yarı düzlemde kararsız öz değer bulunmadığı nümerik olarak hesaplanmıştır. Böylece ele aldığımız ters yönde ilerleyen yanma dalgalarının spektral kararlılı̆̆ ispatlanmıştır.

Anahtar Kelimeler: Yanma dalgaları, Spektrum, Spektral kararlı1ı, Evans fonksiyonu.

\section{Spectral Stability of Traveling Combustion Waves By Evans Function}

\begin{abstract}
In this research combustion waves that appears in a model of enhanced oil recovery by injecting air into a porous media which has some initial fuel are studied. Combustion model involves three dependent variables temperature, oxygen and fuel. Speed of temperature and oxygen are assumed same and counterflow combustion waves are considered. The model is linearized at a counterflow combustion wave and the essential spectrum of the operator of linearized system is investigated. In order to have the spectral stability, a weight function is determined to move the essential spectrum to the left half plane. Evans function is utilized to find point spectrum. Numerical calculation is performed to show that no unstable eigenvalues in the right half plane. Therefore spectral stability of counterflow combustion waves is proved.
\end{abstract}

Keywords: Combustion waves, Spectrum, Spectral stability, Evans function.

\footnotetext{
${ }^{1}$ Harran Üniversitesi, Matematik Bölümü Fen Edebiyat Fakültesi Şanlıurfa, Türkiye, fozbag@harran.edu.tr

${ }^{2}$ Harran Üniversitesi, Matematik Bölümü Fen Edebiyat Fakültesi Şanlıurfa, Türkiye, brc-burcu0123@hotmail.com

${ }^{1}$ http://orcid.org/ 0000-0002-5456-4261 ${ }^{2}$ http://orcid.org/ 0000-0001-5839-8128
} 


\section{Giriş}

Yanma, yakıtın ateşleme sonucu oksijenle birleşmesi ve sıcaklığın ortaya çıkmasıyla oluşan kimyasal bir olaydır. Yanma dalgaları petrol çıkarma yöntemlerinde kullanılan bir metottur. Yanma dalgalarının kararlılığını bilmek, petrol kazanımını en üst düzeye çıkarmaya yardımcı olur. Çalışmamızda hava enjekte edilerek geliştirilmiş petrol geri kazanımı modelinde ortaya çıkan yanma dalgaları incelenmiştir.

Sistem başlangıçta bir miktar katı yakıt bulunan gözenekli bir ortama hava enjekte edilmesi ile oluşan yanma modelini ele alır. Model ilk olarak Akkutlu ve Yortsos tarafından tanıtılmıştır (Akkutlu ve Yortsos, 2003). Model genişletilerek yanma dalgalarının varlığı üzerine daha fazla çalışmalar yapılmıştır (Chapiro ve ark., 2012; Chapiro ve ark., 2014; Chapiro ve Senos, 2017; Ozbag ve ark., 2018; Barlas, 2020). Bu yanma modelimiz sıcaklık, oksijen ve yakıt olmak üzere üç bağımlı değişkenden oluşmaktadır. Sistemimizde sıcaklık denkleminde difüzyon terimi var iken diğer denklemlerde difüzyon terimi yoktur. Bu yanma modeli kısmen parabolik bir sistemdir ve oksijenin difüzyonu görmezden gelinir. Ayrıca katı yakıtın da yayılmayacağı aşikârdır.

Oksijen ve ısının aynı hızda taşındığı durumda yanma dalgalarının varlığı gösterilmiştir (Chapiro ve ark., 2014). Oksijenin sıcaklıktan daha hızlı taşındığı durum için yanma dalgalarının varlığg kanıtlanmıştır (Ozbag ve ark., 2018). Ayrıca bu yanma dalgalarının lineer ve lineer olmayan kararlılı̆̆ı ispatlanmıştır (Ozbag ve Schecter, 2018). Bahsedilen çalışmalarda yanma dalgasının hızı pozitif kabul edilmiştir. Sistemimizde sıcaklık ve oksijenin hızı aynı kabul edilip yanma dalgasının hızı negatif alınarak ters yönde ilerleyen yanma dalgaları göz önünde bulundurulmuştur.

$\mathrm{Bu}$ çalışmada, Chapiro ve Senos'un üzerinde çalıştığı yanma dalgalarının spektral kararlılığı incelenmiştir (Chapiro ve Senos, 2017). Önce bir yanma dalgası civarında sistem linerize edilmiştir. Daha sonra Fourier dönüşümü kullanılarak esas spektrum belirlenmiştir. Ters yönde ilerleyen iki yanma dalgası olduğu açıkça belirtilip, bu yanma dalgalarının sağ ve sol durumlarındaki öz değerleri belirlenmiştir. Elde edilen esas spektrumların grafikleri çizilmiş ve sanal eksene dokunan paraboller oluştuğu gözlemlenmiştir. Böylece esas spektrumun marjinal kararlılığa sahip olduğu görülmüştür, spektral kararlılığı sağlamak için spektrumu sol yarı düzleme taşıyan bir ağırlık fonksiyonu elde edilmiştir. Spektrumun sol yarı düzleme taşınması sonucu spektral kararlılığa sahip olduğunu belirleyebilmek için Evans fonksiyonundan yararlanılmıştır (Gubernov ve ark., 2003).

Evans fonksiyonu, ilerleyen dalgaların kararlılığının belirlenmesi için önemli bir araçtır. Doğrusallaştırılmış operatörün dalga civarındaki spektral analizi için hem analitik hem de nümerik hesaplama açısından kullanışlıdır. Evans fonksiyonu hesaplaması, doğrusal operatörün ayrık spektrumunu bulmaya yani herhangi bir kararsız öz değerinin belirlenmesine yardımcı olur. Bu da 
belirli bir dalganın spektral kararlılığını sağlamaya ve model parametreleri değiştikçe çatallanma noktalarını (kararlılık kaybı) tanımlamaya izin verir (Barker ve ark., 2017).

Bölüm 1' de giriş yer almaktadır. Bölüm 2'de matematiksel model tanıtılmış, önceki sonuçlar özetlenmiştir. Bölüm 3'te ters yönde ilerleyen yanma dalgalarının spektrumu elde edilmiştir. Sağ ve sol durumlar için öz değerler belirlenmiş, grafikleri analiz edilmiştir. Ters yönde ilerleyen yanma dalgaları için ağırlık fonksiyonu elde edilmiş, Evans fonksiyonu kullanılarak ayrık spektrum incelenmiştir. Bölüm 4'te sonuçlara yer verilmiştir.

\section{Matematiksel Model ve Önceki Çalışmalar}

Yanma dalgalarının oluşabilmesi için yakıtın ateşleme sonucu sıcaklık ve oksijen ile birleşmesi gereklidir. Bu durumda sıcaklık, oksijen ve yakıt birbirine bağımlı değişkenlerdir.

Ele aldığımız sistemde; sıcaklık $(\theta)$, yakıt $(\rho)$ ve oksijen $(Y)$ olmak üzere üç bağımlı değişken vardir:

$$
\begin{aligned}
& \partial_{t} \theta+a \partial_{x} \theta=\partial_{x x} \theta+\rho Y \Phi, \\
& \partial_{t} \rho=-\rho Y \Phi, \\
& \partial_{t} Y+a \partial_{x} Y=-\rho Y \Phi, \\
& \Phi=\left\{\begin{array}{cc}
e^{(-1 / \theta)}, & \theta>0 \\
0, & \theta \leq 0 .
\end{array}\right.
\end{aligned}
$$

Sistemdeki sıcaklık ve oksijenin a > 0 hızıyla taşındığı kabul edilmiştir. Aynı zamanda $\Phi$ birim tepki oranı olarak ele alınmıştır. Yanmanın belirli bir tutuşma sıcaklığının üzerinde meydana geldiği varsayılmıştır. Çalışmamızdaki ateşleme sıcaklığı $\theta=0$ olacak şekilde normalize edilmiş olup oksijen difüzyonu göz ardı edilmiştir. Denklemler, parametre sayısını azaltmak için boyutsuzlaştırılmıştır (Ozbag, 2016). Bütün durumlarda $\rho \geq 0$ ve $Y \geq 0$ çözümleri ile ilgilenilmiştir. (1)-(3) denklemleri için $-\infty<\mathrm{x}<+\infty$, $\mathrm{t}>0$ üzerinde sabit sınır koşulları; $(\theta, \rho, Y)(-\infty, \mathrm{t})=$ $\left(\theta^{-}, \rho^{-}, Y^{-}\right)$ve $(\theta, \rho, Y)(+\infty, t)=\left(\theta^{+}, \rho^{+}, Y^{+}\right)$şeklindedir.

Yanma olayının gerçekleşmesi ve yanmanın devam etmesi için ortamda yeteri kadar oksijenin bulunması gerekir. Oksijenin eksik olması durumunda $(\mathrm{Y}=0)$ yanma reaksiyonu durur. Aynı şekilde yanmanın gerçekleşmesi ve devam etmesi için ortamda yakıt bulunması gerekir. Yakıt eksikliği $(\rho=$ 
0) durumunda yanma reaksiyonu durur. Ayrıca ortamdaki sıcaklık düşük ise $(\theta \leq 0)$ yanma reaksiyonu durur.

Yanmanın sınır koşullarında meydana gelmediği kabul edilmiştir. Yani $\mathrm{x}= \pm \infty$ da aşağıdakilerden en az biri olmalıdır:

- Sıcaklık kontrol (TC): düşük sıcaklıkta yani $(\theta \leq 0)$ olduğu zaman reaksiyon durur.

- Yakıt kontrol (FC): yakıt eksikliği yani $(\rho=0)$ olduğu zaman reaksiyon durur.

- Oksijen kontrol (OC): oksijen eksikliği yani $(\mathrm{Y}=0)$ olduğu zaman reaksiyon durur.

\section{1. Önceki Çalışmalar}

Sol durumu $\left(\theta^{-}, \rho^{-}, Y^{-}\right)$, sağ durum ise $\left(\theta^{+}, \rho^{+}, Y^{+}\right)$olan c hizıyla ilerleyen bir yanma dalgası $\left(\theta^{-}, \rho^{-}, Y^{-}\right) \stackrel{c}{\rightarrow}\left(\theta^{+}, \rho^{+}, Y^{+}\right)$şeklinde gösterilir. Dalganın son durumlarında, (1)-(3) denklemlerindeki reaksiyon terimleri ortadan kaybolur. Reaksiyon terimlerinin ortadan kalktığı durumlar TC, FC, OC,

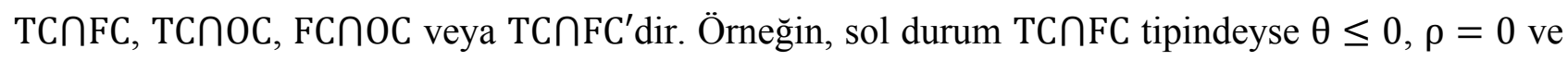
Y değeri pozitiftir.

Bu makalede çalışılan yanma modeli ters yönde ilerlediğinden $\mathrm{c}<0$ kabul edilir. Ayrıca FC hareketsiz katı yakıt olduğundan sol durumda olma olasılı̆̆ yoktur. Yanma dalgası ve enjekte edilen oksijen ters yöne ilerlediğinden sol durumun OC olması imkânsızdır. Diğer taraftan son durumları aynı tipte bir yanma dalgasının olmadığını görmek kolaydır. Geriye kalan diğer olasılıklar TC $\stackrel{c}{\rightarrow}$ FC ve TC $\stackrel{\text { c }}{\rightarrow}$ OC'dir. Bu yanma dalgalarının varlığı kanıtlanmıştır (Chapiro ve Senos, 2017). Bu durumda $\mathrm{TC} \stackrel{\mathrm{c}}{\rightarrow} \mathrm{FC}$ ve $\mathrm{TC} \stackrel{\mathrm{c}}{\rightarrow}$ OC olmak üzere ters yönde ilerleyen iki yanma dalgası vardır.

2.1.1.Teorem: TC sol durumu $\left(\theta^{-}, \rho^{-}, \mathrm{Y}^{-}\right)$yani $0 \geq \theta^{-}, \rho^{-}>0, \mathrm{Y}^{-}>0$ ve a $>0$ olsun. Ayrica $\theta^{-}+\mathrm{Y}^{-}>0$ olduğunu varsayalım. Hızı c $<0$ ve yanma dalgası $\left(\theta^{-}, \rho^{-}, \mathrm{Y}^{-}\right) \stackrel{\mathrm{c}}{\rightarrow}\left(\theta^{+}, \rho^{+}, \mathrm{Y}^{+}\right)$olacak şekilde bir $\left(\theta^{+}, \rho^{+}, \mathrm{Y}^{+}\right)$sağ durumu vardır. Bu durumda $\theta^{+}>0$ ve $\rho^{+}, \mathrm{Y}^{+}$ya da her ikisi de 0 olur. Ayrica $\theta^{+}+\mathrm{Y}^{+}=\theta^{-}+\mathrm{Y}^{-}$ve $\mathrm{c}=\frac{\mathrm{aY^{+ } - \mathrm { aY }}}{\mathrm{Y}^{+}-\mathrm{Y}^{-}+\rho^{-}-\rho^{+}}$.

Bir sonraki bölümde bu iki ters yönde ilerleyen yanma dalgasının spektral kararlılığı incelenecektir.

\section{Ters Yönde İlerleyen Yanma Dalgalarının Spektral Kararlılı̆̆ı}

Bu bölümde, sistem (1)-(3) ters yönde ilerleyen bir yanma dalgası üzerinde linerize edilmiş ve linerize edilmiş operatör $\mathcal{L}^{\prime}$ nin spektrumu incelenmiştir. $\mathcal{L}^{\prime}$ nin spektrumu $\operatorname{Sp}(\mathcal{L})$ olarak ifade edilir, 
ayrık spektrumdan $\operatorname{Sp}_{d}(\mathcal{L})$ ve esas spektrumdan $\operatorname{Sp}_{\text {ess }}(\mathcal{L})$ oluşur. Ayrık spektrum $\mathcal{L}^{\prime}$ nin bütün öz değerlerinden ve bunların sonlu sayıda katlarından oluşan spektrumdur. Spektrumun geri kalanı da esas spektrumu oluşturur. Bu bölümde esas spektrum yani $\operatorname{Sp}_{\text {ess }}(\mathcal{L})$ ele alınmıştır.

(1)-(3) denklemlerinde hareketli koordinata geçmek için $\xi=x-$ ct dönüşümü yapılırsa;

$$
\begin{aligned}
& \partial_{t} \theta=\partial_{\xi \xi} \theta+(c-a) \partial_{\xi} \theta+F, \\
& \partial_{t} \rho=c \partial_{\xi} \rho-F, \\
& \partial_{t} Y=(c-a) \partial_{\xi} Y-F,
\end{aligned}
$$

ve $\mathrm{F}=\rho Y \Phi$ elde edilir. c hızıyla ilerleyen $\mathrm{D}^{*}(\xi)=\left(\theta^{*}(\xi), \rho^{*}(\xi), \mathrm{Y}^{*}(\xi)\right)$ dalgası $(6)-(8)$ 'in değişmez çözümüdür ve $\lim _{\xi \rightarrow-\infty} D^{*}(\xi)=D^{-}=\left(\theta^{-}, \rho^{-}, Y^{-}\right), \lim _{\xi \rightarrow+\infty} D^{*}(\xi)=D^{+}=\left(\theta^{+}, \rho^{+}, Y^{+}\right)$.

$\mathrm{D}^{*}(\xi)$ 'nin $\mathrm{D}^{ \pm}$ya üstel oranda yaklaştığını varsayıyoruz. (6)-(8) denklemlerini $\mathrm{D}^{*}(\xi)$ 'de linerize edersek;

$$
\begin{aligned}
& \partial_{t} \tilde{\theta}=\partial_{\xi \xi} \tilde{\theta}+(c-a) \partial_{\xi} \tilde{\theta}+F_{\theta}\left(D^{*}(\xi)\right) \tilde{\theta}+F_{\rho}\left(D^{*}(\xi)\right) \tilde{\rho}+F_{Y}\left(D^{*}(\xi)\right) \tilde{Y}, \\
& \partial_{t} \tilde{\rho}=c \partial_{\xi} \tilde{\rho}-F_{\theta}\left(D^{*}(\xi)\right) \tilde{\theta}-F_{\rho}\left(D^{*}(\xi)\right) \tilde{\rho}-F_{Y}\left(D^{*}(\xi)\right) \tilde{Y} \\
& \partial_{t} \tilde{Y}=(c-a) \partial_{\xi} \tilde{Y}-F_{\theta}\left(D^{*}(\xi)\right) \tilde{\theta}-F_{\rho}\left(D^{*}(\xi)\right) \tilde{\rho}-F_{Y}\left(D^{*}(\xi)\right) \tilde{Y}
\end{aligned}
$$

elde edilir. (9)-(11) denklemlerini $A_{t}=\mathcal{L} A$ şeklinde yazarak bir matris operatörü elde ederiz;

$$
\mathcal{L}=\left(\begin{array}{ccc}
\partial_{\xi \xi}+(c-a) \partial_{\xi}+F_{\theta}\left(D^{*}(\xi)\right) & F_{\rho}\left(D^{*}(\xi)\right) & F_{Y}\left(D^{*}(\xi)\right) \\
-F_{\theta}\left(D^{*}(\xi)\right) & c \partial_{\xi}-F_{\rho}\left(D^{*}(\xi)\right) & -F_{Y}\left(D^{*}(\xi)\right) \\
-F_{\theta}\left(D^{*}(\xi)\right) & -F_{\rho}\left(D^{*}(\xi)\right) & (c-a) \partial_{\xi}-F_{Y}\left(D^{*}(\xi)\right)
\end{array}\right)
$$

(6)-(8) denklemlerinin $\mathrm{D}^{ \pm}$'da linerize edilmesiyle birbiriyle ilişkili $A_{t}=\mathcal{L}^{ \pm} A$ şeklinde sabit katsayılı doğrusal kısmi diferansiyel denklemler vardır. $\mathcal{L}^{2}$ uzayında $\mathcal{L}^{ \pm}$'nin spektrumu Fourier dönüşümü kullanılarak hesaplanır: 


$$
\hat{\mathcal{L}}^{ \pm}=\left(\begin{array}{ccc}
-\mu^{2}+i \mu(c-a)+F_{\theta}\left(D^{ \pm}\right) & F_{\rho}\left(D^{ \pm}\right) & F_{Y}\left(D^{ \pm}\right) \\
-F_{\theta}\left(D^{ \pm}\right) & i \mu c-F_{\rho}\left(D^{ \pm}\right) & -F_{Y}\left(D^{ \pm}\right) \\
-F_{\theta}\left(D^{ \pm}\right) & -F_{\rho}\left(D^{ \pm}\right) & i \mu(c-a)-F_{Y}\left(D^{ \pm}\right)
\end{array}\right)
$$

$\mathcal{L}^{\prime}$ in temel spektrumunun sağ taraftaki sınırı $\operatorname{Sp}\left(\mathcal{L}^{-}\right)$ve $\operatorname{Sp}\left(\mathcal{L}^{+}\right)$'nin sağ sınırının birleşimidir.

3.1.Tanım: Eğer $\mathcal{L}^{ \pm}$' nin spektrumu

- $\quad \mu>0$ için $\{\operatorname{Re} \lambda<-\mu\}$ yarı düzlemi içinde yer alıyorsa, bu durumda $\mathrm{D}^{ \pm}$kararlıdır.

- $\{\operatorname{Re} \lambda \leq 0\}$ yarı düzlemi içinde yer alıyorsa ve sanal eksene dokunuyorsa, bu durumda $\mathrm{D}^{ \pm}$ marjinal olarak kararlıdir.

- $\operatorname{Re} \lambda>0$ noktalarını içeriyorsa, bu durumda $\mathrm{D}^{ \pm}$kararsızdır.

3.2.Tanım: $D^{*}(\xi)$ ilerleyen dalgası aşağıdaki şartları sağlarsa $X$ uzayında spektral olarak kararlıdır.

- X uzayında, $0 \mathcal{L}^{\prime}$ nin izole edilmiş basit bir öz değeridir ve buna karşılık gelen öz fonksiyon $\mathrm{D}^{*}(\xi)^{\prime}$ 'dir.

- $\mu>0$ için $\mathcal{L}^{\prime}$ nin spektrumunun geri kalanı $\operatorname{Re} \lambda<-\mu$ içinde yer alır.

\subsection{Ters Yönde İlerleyen Dalga Denklemlerinin Spektrumu}

Ters yönde ilerleyen iki yanma dalgası vardır: $\mathrm{TC} \stackrel{\mathrm{c}}{\rightarrow} \mathrm{FC}$ ve $\mathrm{TC} \stackrel{\mathrm{c}}{\rightarrow}$ OC. Her iki yanma dalgası için TC'yi sol durumda; FC ve OC'yi sağ durumda inceleriz.

\subsubsection{TC Sol Durumu}

$\left(\theta^{-}, \rho^{-}, Y^{-}\right)$'da $0 \geq \theta^{-}, \rho^{-}>0, Y^{-}>0$ şeklindedir. Ayrıca modelin tanımındaki $F=\rho Y \Phi$ ifadesinden ve $0 \geq \theta^{-}$olduğundan $\Phi=0$ dir. Bu da bize;

$F_{\theta}\left(D^{-}\right)=0, \quad F_{\rho}\left(D^{-}\right)=0, \quad F_{Y}\left(D^{-}\right)=0$ eşitliklerini verir. Elde ettiğimiz ifadeleri (13) denkleminde yerine yazarsak:

$$
\hat{\mathcal{L}}^{-}=\left(\begin{array}{ccc}
-\mu^{2}+i \mu(c-a) & 0 & 0 \\
0 & i \mu c & -0 \\
0 & 0 & i \mu(c-a)
\end{array}\right)
$$


elde edilir. $\hat{\mathcal{L}}^{- \text {' } n i n ~ s p e k t r u m u ~} \mathbb{R}^{\prime}$ deki bazı $\mu$ 'ler için (14) denkleminin öz değerleri olan $\lambda$ 'ların kümesi aşağıdaki şekildedir:

$$
\lambda(\mu)=-\mu^{2}+i \mu(c-a), \lambda(\mu)=i \mu c \text { ve } \lambda(\mu)=i \mu(c-a) .
$$

Şekil 1' de bu parametrik öz değerlerin grafiği görülmektedir. Sol yarı düzlemde sanal eksene teğet orijinden geçen bir paraboldür. Ayrıca sanal eksenin üzerinde parabole teğet iki dikey doğruya sahiptir.

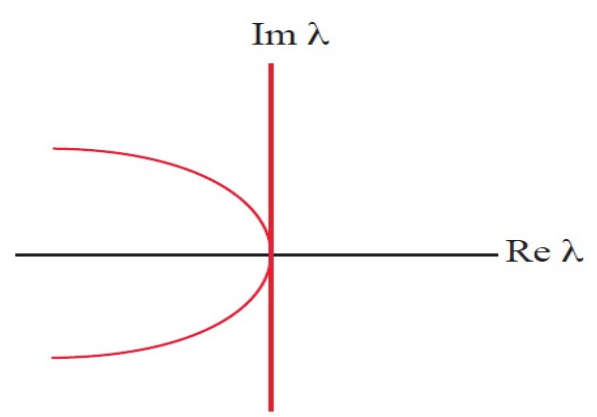

\subsubsection{OC Să̆ Durumu}

$\left(\theta^{+}, \rho^{+}, \mathrm{Y}^{+}\right)^{\prime} \mathrm{da} \theta^{+}>0, \rho^{+}>0, \mathrm{Y}^{+}=0$ şeklindedir. Ayrıca modelin tanımındaki $\mathrm{F}=\rho \mathrm{Y} \Phi$ ifadesinden; $F_{\theta}\left(D^{+}\right)=0, F_{\rho}\left(D^{+}\right)=0, F_{Y}\left(D^{+}\right)=\rho^{+} \Phi\left(\theta^{+}\right)$eşitliklerini verir. Elde ettiğimiz ifadeleri (13) denkleminde yerine yazarsak:

$$
\hat{\mathcal{L}}^{+}=\left(\begin{array}{ccc}
-\mu^{2}+i \mu(c-a) & 0 & \rho^{+} \Phi\left(\theta^{+}\right) \\
0 & i \mu c & -\rho^{+} \Phi\left(\theta^{+}\right) \\
0 & 0 & i \mu(c-a)-\rho^{+} \Phi\left(\theta^{+}\right)
\end{array}\right)
$$

elde edilir. $\hat{\mathcal{L}}^{+}$'nin spektrumu $\mathbb{R}^{\prime}$ deki bazı $\mu^{\prime}$ ler için (15) denkleminin öz değerleri olan $\lambda$ 'ların kümesi aşağıdaki şekildedir: 


$$
\lambda(\mu)=-\mu^{2}+i \mu(c-a), \lambda(\mu)=i \mu c \text { ve } \lambda(\mu)=i \mu(c-a)-\rho^{+} \Phi\left(\theta^{+}\right) .
$$

Şekil 2' de bu parametrik öz değerlerin grafiği görülmektedir. Sol yarı düzlemde sanal eksene teğet orijinden geçen bir paraboldür. Ayrıca sanal eksenin üzerinde parabole teğet ve sol yarı düzlemde parabolün üzerinden geçen iki dikey doğruya sahiptir.

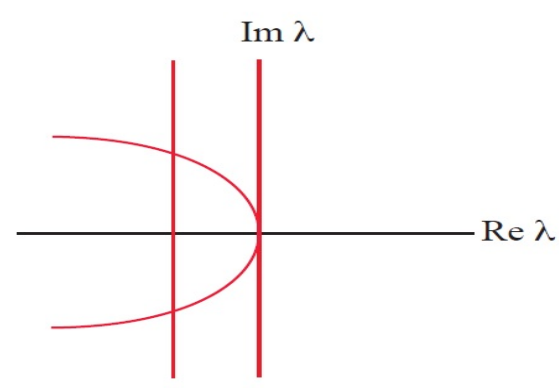

\subsubsection{FC Să̆ Durumu}

$\left(\theta^{+}, \rho^{+}, \mathrm{Y}^{+}\right)$'da $\theta^{+}>0, \rho^{+}=0, \mathrm{Y}^{+}>0$ şeklindedir. Ayrıca modelin tanımındaki $\mathrm{F}=\rho \mathrm{Y} \Phi$ ifadesinden; $F_{\theta}\left(D^{+}\right)=0, F_{\rho}\left(D^{+}\right)=Y^{+} \Phi\left(\theta^{+}\right), F_{Y}\left(D^{+}\right)=0$ eşitliklerini verir. Elde ettiğgimiz ifadeleri (13) denkleminde yerine yazarsak:

$$
\hat{\mathcal{L}}^{+}=\left(\begin{array}{ccc}
-\mu^{2}+i \mu(c-a) & Y^{+} \Phi\left(\theta^{+}\right) & 0 \\
0 & i \mu c-Y^{+} \Phi\left(\theta^{+}\right) & 0 \\
0 & Y^{+} \Phi\left(\theta^{+}\right) & i \mu(c-a)
\end{array}\right)
$$

elde edilir. $\hat{\mathcal{L}}^{+}$' nin spektrumu $\mathbb{R}^{\prime}$ deki bazı $\mu^{\prime}$ ler için (16) denkleminin öz değerleri olan $\lambda$ 'ların kümesi aşağıdaki şekildedir:

$$
\lambda(\mu)=-\mu^{2}+i \mu(c-a), \lambda(\mu)=i \mu c-Y^{+} \Phi\left(\theta^{+}\right) \text {ve } \lambda(\mu)=i \mu(c-a) .
$$

Grafiği Şekil 2 ile aynıdır. 
Tüm durumlar için $\operatorname{Sp}\left(\mathcal{L}^{-}\right)$ve $\operatorname{Sp}\left(\mathcal{L}^{+}\right)$sanal eksene dokunduğundan spektral kararlılığa sahip değiliz. Eğer bu spektrumları sanal eksenin soluna taşıyabilecek bir ağırlık fonksiyonu bulanabilinirse spektral kararlılık elde edilebilir.

\subsection{Ters Yönde İlerleyen Yanma Dalgaları İçin Ağırlık Fonksiyonu}

$$
\begin{aligned}
& \alpha=\left(\alpha_{-}, \alpha_{+}\right) \in \mathbb{R}^{2} \text { için } \Upsilon_{\alpha}: \mathbb{R} \rightarrow \mathbb{R} \text { bir ağırlık fonksiyonu olsun: } \\
& \Upsilon_{\alpha}(\xi):= \begin{cases}e^{\alpha_{-} \xi}, & \xi \leq 0 \\
e^{\alpha_{+} \xi}, & \xi \geq 0 .\end{cases}
\end{aligned}
$$

$\chi_{0}, L^{2}\left(\mathbb{R}, \mathbb{R}^{3}\right)$ veya $H^{1}\left(\mathbb{R}, \mathbb{R}^{3}\right)$ standart Banach uzaylarından biri olsun ve \|\|$_{0}$ normuna sahip olsun. $\chi_{\alpha}, \Upsilon_{\alpha}(\xi)$ ağırlık fonksiyonu ile bir ağırlık uzayı belirtsin. Yani $x(\xi) \epsilon \chi_{\alpha}$ için, $\Upsilon_{\alpha}(\xi) x(\xi) \epsilon \chi_{0}$ ve $\|\mathrm{x}\|_{\alpha}=\left\|\Upsilon_{\alpha}(\xi) \mathrm{x}(\xi)\right\|_{0}$ olur.

$\mathcal{L}^{\prime}$ nin spektrumunu $\chi_{\alpha}$ üzerinde bir operatör olarak incelemek için $\chi=(\tilde{\theta}(\xi), \widetilde{\rho}(\xi)$, $\tilde{Y}(\xi)) \epsilon \chi_{\alpha}$ ve $\mathcal{W}=\Upsilon_{\alpha}(\xi) \chi=(u(\xi), v(\xi), z(\xi)) \epsilon \chi_{0}$ alalım.

$\mathcal{W}=\Upsilon_{\alpha} \chi$ ifadesi $\Upsilon_{\alpha}{ }^{-1}$ ile çarpılırsa $\chi=\Upsilon_{\alpha}{ }^{-1} \mathcal{W}$ elde edilir. $\chi_{\mathrm{t}}=\mathcal{L} \chi$ denkleminde $\chi$ yerine yazılırsa $\Upsilon_{\alpha}{ }^{-1} \mathcal{W}_{\mathrm{t}}=\mathcal{L} \Upsilon_{\alpha}{ }^{-1} \mathcal{W}$ elde edilir. $\Upsilon_{\alpha}{ }^{-1} \mathcal{W}_{\mathrm{t}}=\mathcal{L} \Upsilon_{\alpha}{ }^{-1} \mathcal{W}$ ifadesi $\Upsilon_{\alpha}$ ile çarp1lırsa $\mathcal{W}_{\mathrm{t}}=$ $\Upsilon_{\alpha} \mathcal{L} \Upsilon_{\alpha}{ }^{-1} \mathcal{W}$ elde edilir. Burada $\Upsilon_{\alpha} \mathcal{L} \Upsilon_{\alpha}{ }^{-1}$ operatörü $\chi_{0}$ üzerinde bir lineer operatördür. L'nin $\chi_{\alpha}$ 'daki spektrumunu bulmak yerine, $\mathcal{L}_{\alpha}=\Upsilon_{\alpha} \mathcal{L} \Upsilon_{\alpha}{ }^{-1}$ izomorfik operatörün spektrumu $\chi_{0}$ üzerinde bulunur. $\quad \mathcal{W}=\Upsilon_{\alpha}(\xi) \chi=e^{\alpha \xi} \chi \quad$ olduğundan $\quad(u(\xi), v(\xi), z(\xi))=e^{\alpha \xi}(\tilde{\theta}(\xi), \widetilde{\rho}(\xi), \tilde{Y}(\xi)) '$ dir. $\quad$ Bu durumda;

$$
\mathcal{L}_{\alpha}=\left(\begin{array}{ccc}
\partial_{\xi \xi}+\left(c-a-2 \alpha_{ \pm}\right) \partial_{\xi} & F_{\rho}\left(D^{*}\right) & F_{Y}\left(D^{*}\right) \\
+\alpha_{ \pm}{ }^{2}+a \alpha_{ \pm}-c \alpha_{ \pm}+F_{\theta}\left(D^{*}\right) & & \\
-F_{\theta}\left(D^{*}\right) & c \partial_{\xi}-c \alpha_{ \pm}-F_{\rho}\left(D^{*}\right) & -F_{Y}\left(D^{*}\right) \\
-F_{\theta}\left(D^{*}\right) & -F_{\rho}\left(D^{*}\right) & (c-a)\left(\partial_{\xi}-\alpha_{ \pm}\right)-F_{Y}\left(D^{*}\right)
\end{array}\right)
$$

elde edilir. $\mathcal{W}_{\mathrm{t}}=\mathcal{L}_{\alpha} \mathcal{W}$ denkleminde $\xi \rightarrow \pm \infty$ alınırsa $\mathcal{W}_{t}=\mathcal{L}_{\alpha}{ }^{ \pm} \mathcal{W}$ sabit katsayılı lineer diferansiyel denklem elde edilir. Burada $\mathcal{L}_{\alpha} \pm$ 


$$
\left(\begin{array}{ccc}
\partial_{\xi \xi}+\left(c-a-2 \alpha_{ \pm}\right) \partial_{\xi} & F_{\rho}\left(D^{ \pm}\right) & F_{Y}\left(D^{ \pm}\right) \\
+\alpha_{ \pm}{ }^{2}+a \alpha_{ \pm}-c \alpha_{ \pm}+F_{\theta}\left(D^{ \pm}\right) & & \\
-F_{\theta}\left(D^{ \pm}\right) & c \partial_{\xi}-c \alpha_{ \pm}-F_{\rho}\left(D^{ \pm}\right) & -F_{Y}\left(D^{ \pm}\right) \\
-F_{\theta}\left(D^{ \pm}\right) & -F_{\rho}\left(D^{ \pm}\right) & (c-a)\left(\partial_{\xi}-\alpha_{ \pm}\right)-F_{Y}\left(D^{ \pm}\right)
\end{array}\right)
$$

şeklindedir. $\mathcal{L}_{\alpha}$ esas spektrumunun sağ taraftaki $\operatorname{sinırı~} \mathrm{Sp}_{\mathcal{L}_{\alpha}}{ }^{-}$ve $\mathrm{Sp} \mathcal{L}_{\alpha}{ }^{+}$nın sağ taraftaki sınırlarının birleşimidir. Bu spektrumlar $\mathrm{L}^{2}$ veya $\mathrm{H}^{1}$ 'de aynıdır. Bu yüzden Fourier dönüşümü kullanarak $\mathrm{L}^{2}$ 'de hesapliyoruz.

\subsubsection{TC Sol Durumu}

Benzer hesaplama ile

$$
\mathcal{L}_{\alpha}{ }^{-}=\left(\begin{array}{ccc}
-\mu^{2}+i \mu\left(c-a-2 \alpha_{-}\right) & 0 & 0 \\
+\left(\alpha_{-}\right)^{2}+(a-c) \alpha_{-} & & \\
0 & i \mu c-c \alpha_{-} & -0 \\
0 & 0 & i \mu(c-a)-(c-a) \alpha_{-}
\end{array}\right)
$$

bulunur. $\mathcal{L}_{\alpha}{ }^{-}$'nin spektrumu $\mathbb{R}^{\prime}$ deki bazı $\mu^{\prime}$ ler için (19) denkleminin öz değerleri olan $\lambda$ 'ların kümesi aşağıdaki şekildedir:

$$
\begin{aligned}
& \lambda(\mu)=-\mu^{2}+i \mu\left(c-a-2 \alpha_{-}\right)+\left(\alpha_{-}\right)^{2}+(a-c) \alpha_{-}, \\
& \lambda(\mu)=i \mu c-c \alpha_{-}, \\
& \lambda(\mu)=i \mu(c-a)-(c-a) \alpha_{-} .
\end{aligned}
$$

(14)'deki spektrumu sanal eksenin soluna taşımamız için tüm öz değerlerin reel kısmının negatif olması gerekir. Bu durumda;

$$
-\mu^{2}+\left(\alpha_{-}\right)^{2}+(a-c) \alpha_{-}<0,-c \alpha_{-}<0,-(c-a) \alpha_{-}<0 \text { ifadelerinden } \alpha_{-}<0 \text { ve } \alpha_{-}>
$$
$c-a$ bulunur. Düzenlendiğinde $c-a<\alpha_{-}<0$ elde edilir. Elde edilen ağırlık fonksiyonuyla spektrum sol yarı düzleme taşındığında Şekil 3 elde edilir. 


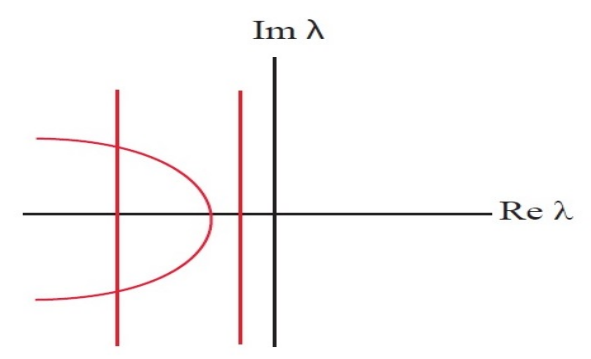

Şekil 3. Spektrumların ağırlık fonksiyonu ile sanal eksenin soluna taşınmış hali.

\subsubsection{OC ve FC Sağ Durumları}

OC ve FC sağ durum için de aynı işlemler yapıldığında; $\mathrm{c}-\mathrm{a}<\alpha_{+}<0$ elde edilir. Elde edilen ağırlık fonksiyonuyla spektrum sol yarı düzleme taşındığında yine Şekil 3 elde edilir.

\subsection{Evans Fonksiyonu}

Evans fonksiyonu, $\mathcal{L}$ diferansiyel operatörünün ayrık spektrumunu bulmak için kullanılan bir analitik fonksiyondur (Gubernov ve ark., 2003; Alexander ve ark.,1990; Sandstede, 2002). Basit sistemler için analitik olarak çözülebilir, ancak genel olarak belirli bir PDE için açıkça hesaplamak zordur.

Bu bölümde, ters yönde ilerleyen yanma dalgalarının ayrık spektrumu için Evans fonksiyonun sayısal sonucu inceleniyor. Yani esas spektrumu kararlı hale getirmek için bulunan $\alpha=\left(\alpha_{-}, \alpha_{+}\right) \in \mathbb{R}^{2}$ için c $-\mathrm{a}<\alpha_{ \pm}<0$ olmak üzere $\mathcal{L}_{\alpha}$ operatörünün ayrık spektrumu incelenecektir. Evans fonksiyonu $D(\lambda)$, esas spektrumun sağında tanımlanan analitik bir fonksiyondur, $\mathcal{L}_{\alpha}$ 'nın özdeğeri için 0'a eşittir. Karmaşık düzlemde kapalı bir C eğrisi üzerinde $D(\lambda)$ grafiği çizilerek, sıfırdaki sarmal sayısı $\mathcal{L}_{\alpha}$ 'nın $\mathrm{C}$ deki öz değerlerinin sayısına eşit olan kapalı bir $\mathrm{D}(\mathrm{C})$ eğrisi elde edilir. İyi seçilmiş eğri, sağ yarı düzlemde $\mathcal{L}_{\alpha}$ 'nın tüm öz değerlerini vermelidir. Bu durumda C'nin boyutunu belirli bir noktadan sonra artırmanın ek öz değerler sağlamayacağı aşikârdır. C'yi yeterince büyük seçerek sağ yarı düzlemde ek öz değerler olmadığı gösterilir. $\mathcal{W}_{t}=\mathcal{L}_{\alpha}{ }^{ \pm} \mathcal{W}$ denkleminin öz değer problemi yazılırsa:

$$
\left\{\begin{array}{c}
\lambda u=u_{\xi \xi}+\left(c-a-2 \alpha_{ \pm}\right) u_{\xi}+\left(\alpha_{ \pm}^{2}+a \alpha_{ \pm}-c \alpha_{ \pm}\right) u+F_{\theta}\left(D^{*}\right) u+F_{\rho}\left(D^{*}\right) v+F_{Y}\left(D^{*}\right) z \\
\lambda v=c v_{\xi}-c \alpha_{ \pm} v-F_{\theta}\left(D^{*}\right) u-F_{\rho}\left(D^{*}\right) v-F_{Y}\left(D^{*}\right) z \\
\lambda z=(c-a) z_{\xi}-(c-a) \alpha_{ \pm} Z-F_{\theta}\left(D^{*}\right) u-F_{\rho}\left(D^{*}\right) v-F_{Y}\left(D^{*}\right) z
\end{array}\right.
$$


elde edilir. $\mathrm{w}=\mathrm{u}_{\xi}$ alarak (20) sistemi $\lambda$ parametresiyle birinci dereceden bir sistem olarak yeniden yazilirsa

$$
\begin{aligned}
& u_{\xi}=w \\
& w_{\xi}=\lambda u-\left(c-a-2 \alpha_{ \pm}\right) w-\left(\alpha_{ \pm}{ }^{2}+a \alpha_{ \pm}-c \alpha_{ \pm}\right) u-F_{\theta}\left(D^{*}\right) u-F_{\rho}\left(D^{*}\right) v-F_{Y}\left(D^{*}\right) z \\
& v_{\xi}=\frac{1}{c}\left(\lambda v+c \alpha_{ \pm} v+F_{\theta}\left(D^{*}\right) u+F_{\rho}\left(D^{*}\right) v+F_{Y}\left(D^{*}\right) z\right) \\
& z_{\xi}=\frac{1}{c-a}\left(\lambda_{z}+(c-a) \alpha_{ \pm} z+F_{\theta}\left(D^{*}\right) u+F_{\rho}\left(D^{*}\right) v+F_{Y}\left(D^{*}\right) z\right)
\end{aligned}
$$

elde edilir. Bu denklem sistemi $Z_{\xi}=A(\xi, \lambda) Z$ formundadır.

$A_{ \pm}$'nın $\lambda$ cinsinden analitik olan limit matrisinin tanımı; $A_{ \pm}(\lambda)=\lim _{\xi \rightarrow \pm \infty} A(\xi, \lambda)$ 'dır. Ayrıca $A_{-}(\lambda)$ 'nın kararsız alt uzayı $U_{-}(\lambda)$ 'nın boyutu bir ve $A_{+}(\lambda)$ 'nın kararlı alt uzayı $S_{+}(\lambda)$ 'nın boyutu üçtür. Bu da tüm faz uzayının boyutu olan dörtte birleşir. Evans fonksiyonunu $\lambda$ 'da analitik olarak tanımlamak

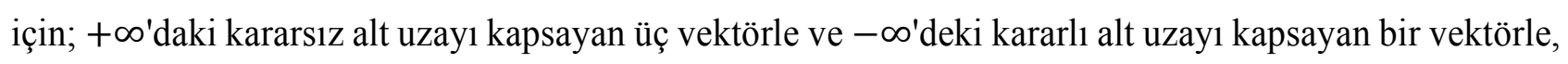
(22) denklemini kullanıp bunları $\xi=0$ 'a doğru hesaplıyoruz. $\xi=0$ için hesaplamalar sonucunda $\mathrm{Z}_{1}{ }^{+}(\xi, \lambda), \mathrm{Z}_{2}{ }^{+}(\xi, \lambda), \mathrm{Z}_{3}{ }^{+}(\xi, \lambda), \mathrm{Z}_{4}{ }^{-}(\xi, \lambda)$ şeklinde dört vektör elde ediyoruz. O halde Evans fonksiyonu şu şekilde tanımlanabilir;

$$
D(\lambda)=\operatorname{det}\left(Z_{1}{ }^{+} Z_{2}{ }^{+} Z_{3}{ }^{+} Z_{4}{ }^{-}\right)
$$

Dolayısıyla, sadece $\mathrm{D}(\lambda)=0$ ise bir özdeğer vardır ve kök sırası, özdeğerin cebirsel çokluğuna karşılık gelir.

Sabit a, $\theta^{-}, \rho^{-}$değerleri için Evans fonksiyonu STABLAB (Barker ve ark., 2009) kullanılarak hesaplanmıştır. (6)-(8) denklemleri verilen sol durum için indirgenirse:

$$
\begin{aligned}
& \dot{\theta}=(a-c)\left(\theta-\theta^{-}\right)-c\left(\rho-\rho^{-}\right) \\
& \dot{\rho}=\left(\frac{\rho-\rho^{-}}{c-a}+\frac{Y^{-}}{c}\right) \rho \Phi(\theta)
\end{aligned}
$$


elde edilir. $\mathrm{Y}^{-}=7$ olarak sabitleyip, $\mathrm{TC} \stackrel{\mathrm{c}}{\rightarrow}$ FC'ye karşılık gelen sağ durumu $\rho^{+}=0$ olan bir c hızı AUTO (Doedel ve ark., 2007) kullanılarak hesaplanmıştır. Başlangıç koşullarına uygun olarak $\mathrm{a}=$ $0.5, \theta^{-}=-0.1$ ve $\rho^{-}=5$ değerleri için $\mathrm{c}=-3.6744$ olarak bulunmuştur. Ayrıca 2.1.1. Teoremi kullanılarak $\theta^{+}=4.3$ olarak elde edilmiştir. (21)-(22) sisteminin nümerik çözümü Şekil 4'te verilmiştir. Şekil 5'te Evans fonksiyonu D(C)'yi göstermektedir. Buradaki C eğrisi dikey yarıçaplı yarım daire; $\left(x+10^{-4}\right)^{2}+y^{2}=25^{2}, x \geq-10^{-4}$ 'tür. 0 civarında eğrinin sarmal sayısının bir olduğu yakınlaştırılmış Şekil 6'da verilmiştir. Bu da $\lambda=0$ öz değeridir ve C eğrisi içinde başka öz değer yoktur.

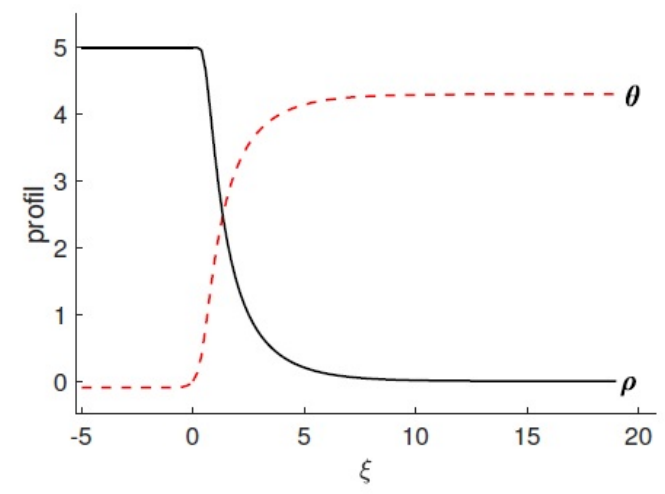

Şekil 4. (21)-(22) sisteminin $\mathrm{a}=0.5, \theta^{-}=-0.1, \mathrm{Y}^{-}=7, \rho^{-}=5$ ve $\mathrm{c}=-3.6744$ için profili.

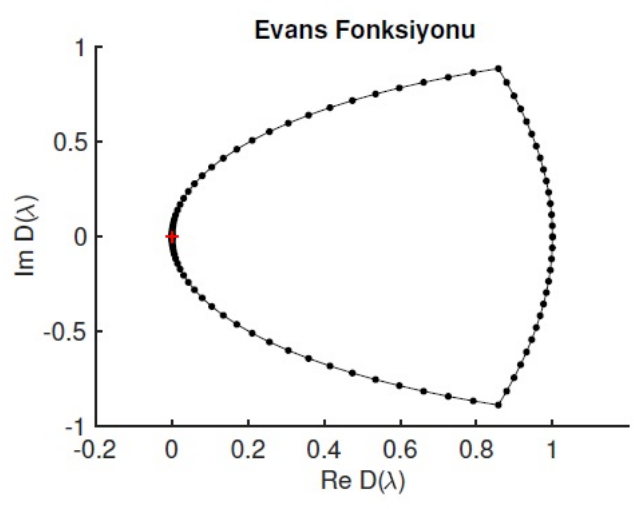

Şekil 5. Yarıçapı 25 olan yarım çember şeklindeki kontur için Evans fonksiyonu 


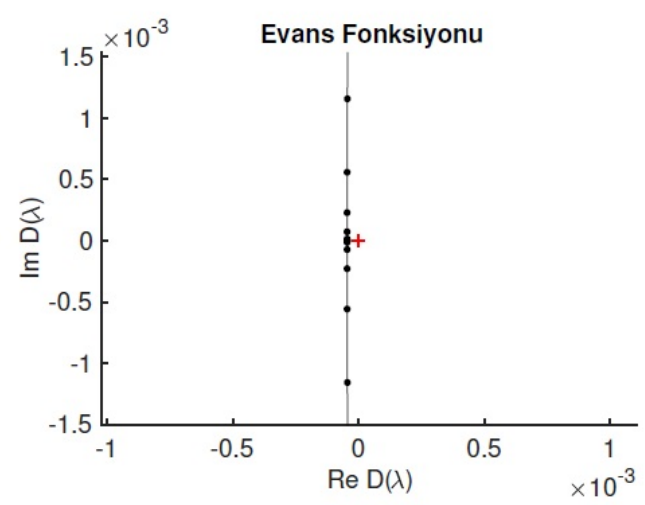

Şekil 6. 0 'ın eğri içinde olduğunu gösteren Şekil 5'in orijin civarında yakınlaştırılmış hali

Benzer şekilde $\mathrm{Y}^{-}=1$ olarak sabitleyip, $\mathrm{TC} \stackrel{\mathrm{c}}{\rightarrow}$ OC' ye karşılık gelen sağ durumu $\mathrm{Y}^{+}=0$ olan bir c hızı AUTO (Doedel ve ark.) kullanılarak hesaplanmıştır. Başlangıç koşullarına uygun olarak aynı $\mathrm{a}=0.5, \theta^{-}=-0.1$ ve $\rho^{-}=5$ değerleri için $\mathrm{c}=-0.44$ olarak bulunmuştur. Ayrıca 2.1.1. Teoremi kullanılarak $\theta^{+}=0.9$ olarak elde edilmiştir. (21)-(22) sisteminin nümerik çözümü Şekil 7'de verilmiştir. Şekil 8' de Evans fonksiyonu D(C)'yi göstermektedir. Buradaki C eğrisi dikey yarıçaplı yarım daire; $\left(x+10^{-4}\right)^{2}+y^{2}=25^{2}, x \geq-10^{-4}$ 'tür. 0 civarında eğrinin sarmal sayısının bir olduğu yakınlaştırılmış Şekil 9'da verilmiştir. $\mathrm{Bu}$ da $\lambda=0$ öz değeridir ve C eğrisi içinde başka öz değer yoktur.

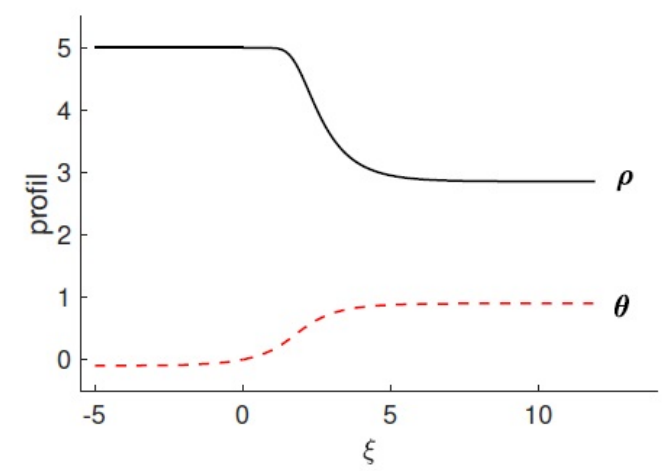




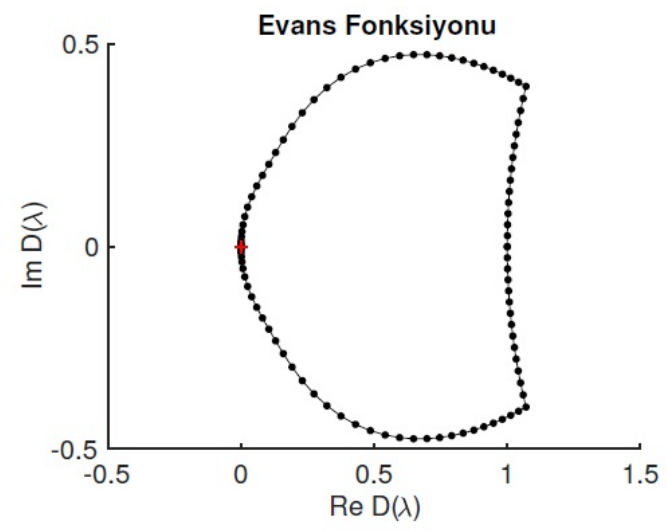

Şekil 8. Yarıçapı 25 olan yarım çember şeklindeki kontur için Evans fonksiyonu

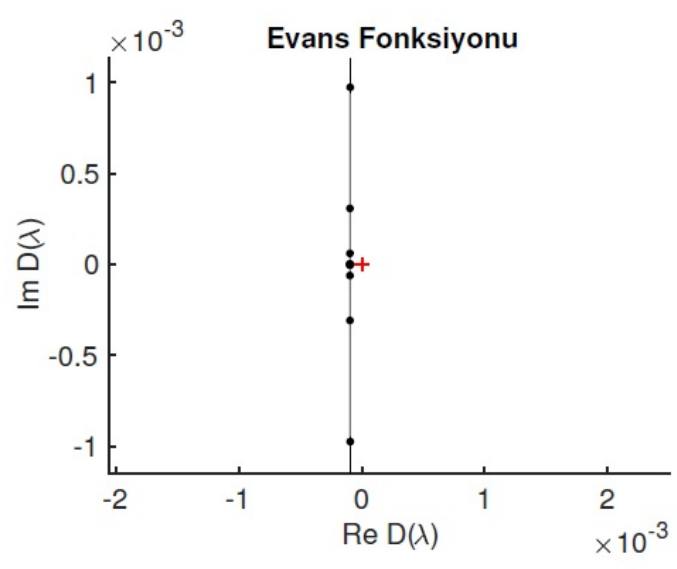

Şekil 9. 0'ın eğri içinde olduğunu gösteren Şekil 8'in orijin civarında yakınlaştırılmış hali

\section{Sonuç}

Bu çalışmada bir miktar yakıt bulunan gözenekli bir ortama hava enjekte edilmesi ile oluşan yanma dalgaları incelenmiştir. Sistemimizde sıcaklık ve oksijenin hızı aynı kabul edilip ters yönde ilerleyen yanma dalgaları göz önünde bulundurulmuştur.

Spektrum, esas spektrum ve ayrık spektrumdan oluşmaktadır. İlk olarak Fourier dönüşümü kullanılarak esas spektrum belirlenmiştir. Ters yönde ilerleyen iki yanma dalgası olduğu açıkça 
belirtilip, bu yanma dalgalarının sağ ve sol durumlardaki öz fonksiyonları belirlenmiştir. Elde edilen öz fonksiyonların grafikleri çizilmiş, sanal eksene dokunan paraboller oluştuğu gözlemlenmiştir. Spektral kararlılığın sağlanması için esas spektrumu sol yarı düzleme taşıyan bir ağırlık fonksiyonu elde edilmiştir.

Ayrık spektrumu bulabilmek için Evans fonksiyonu kullanılmıştır. Bunun için başlangıç koşullarına uygun olarak belirlenen parametreler kullanılarak ters yönde ilerleyen yanma dalgalarının hızı bulunmuştur. Belirlenen yarım daire içinde kalan kararsız öz değerler için Evans fonksiyon grafiği çizilmiştir. Evans fonksiyonunun sarmal sayısının bir olduğu gözlemlenmiştir. Yani orijin noktası haricinde başka bir öz değer bulunmadığı belirlenmiştir. Böylece ele aldığımız ters yönde ilerleyen yanma dalgalarının spektral kararlılığı ispatlanmıştır.

\section{Yazarların Katkısı}

$\mathrm{Bu}$ makale, birinci yazarın danışmanlığında bulunan ikinci yazarın yüksek lisans tezinden üretilmiştir.

\section{Çıkar Çatışması Beyanı}

Yazarlar arasında herhangi bir çıkar çatışması bulunmamaktadır.

\section{Araştırma ve Yayın Etiği Beyanı}

Yapılan çalışmada araştırma ve yayın etiğine uyulmuştur.

\section{Kaynaklar}

Akkutlu, I.Y. ve Yortsos, Y.C. (2003). The Dynamics of in-situ combustion fronts in porous media. Combustion and Flame, 13, 229-247.

Alexander, J., Gardner, R. ve Jones, C. (1990). A topological invariant arising in the stability analsis of traveling waves. J. Reine Angew. Math., 410, 167-212.

Barker, B., Humpherys, J., Lyng, G. ve Lytle, J. (2017). Evans function computation for the stability of travelling waves. Philosophical Transactions Of The Royal Society A, 376(2117), 20170184.

Barker, B., Humpherys, J. ve Zumbrun K. (2009). STABLAB: a MATLAB-based numerical library for Evans function computation. http://www.impact.byu.edu/stablab/.

Barlas, G. (2020). Gözenekli ortamda ters yönde ilerleyen yanma dalgalarının varlı̆̆ı. Yüksek Lisans Tezi, Harran Üniversitesi, Fen Bilimleri Enstitüsü, Şanlıurfa.

Chapiro, G., Mailybaev, A.A., de Souza, A.J., Marchesin, D. ve Bruining, J. (2012). Asymptotic approximation of long-time solution for low-temperature filtration combustion. Computational Geosciences, 16, 799808. 
Chapiro, G., Marchesin, D. ve Schecter, S. (2014). Combustion waves and Riemann solutions in light porous foam. Journal of Hyperbolic Differential Equations, 11, 295-328.

Chapiro, G. ve Senos, L. (2017). Riemann solutions for counterflow combustion in light porous foam. Computational and Applied Mathematics, 37, 1721-1736.

Doedel, E.J., Champneys, A.R., Fairgrieve, T.F., Kuznetsov, Y.A., Oldeman, B., Paffenroth, R., Sandstede, B., Wang, X. ve Zhang, C. (2007). 2007 AUTO-07P: continuation and bifurcation software for ordinary differential equations. Concordia University, Montreal, Canada. http://indy.cs.concordia.ca/auto/.

Gubernov, V., Mercer, G.N., Sidhu, H.S. ve Weber, R.O. (2003). Evans function stability of combustion waves. Society For Industrial and Applied Mathematics, 63, 1259-1275.

Ozbag, F., Schecter, S. ve Chapiro, G. (2018). Traveling waves in a simplified gas-solid combustion model in porous media. Adv. Differential Equations, 23, 409-454.

Ozbag, F. ve Schecter, S. (2018). Stability of conmbustion waves in a simplified gas-solid combustion model in porous media. Philosophical Transactions A, 376(2117), 20170185.

Ozbag, F. (2016). Stability analysis of combustion waves in porous media. Doctoral dissertation, North Carolina State University, USA.

Sandstede, B. (2002). Stability of traveling waves. Handbook of dynamical systems, 2, 983-1055. 